\title{
Microwave characterization of magnetically hard and soft ferrite nanoparticles in K-band
}

Cite as: J. Appl. Phys. 116, 154306 (2014); https://doi.org/10.1063/1.4898138

Submitted: 01 August 2014 . Accepted: 19 September 2014 . Published Online: 20 October 2014

C. Della Pina, E. Falletta, A. M. Ferretti, A. Ponti, G. G. Gentili, V. Verri, and R. Nesti
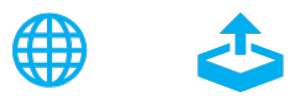

\section{ARTICLES YOU MAY BE INTERESTED IN}

Exchange coupling and microwave absorption in core/shell-structured hard/soft ferritebased $\mathrm{CoFe}_{2} \mathrm{O}_{4} / \mathrm{NiFe}_{2} \mathrm{O}_{4}$ nanocapsules

AIP Advances 7, 056403 (2017); https://doi.org/10.1063/1.4972805

A review and analysis of microwave absorption in polymer composites filled with carbonaceous particles

Journal of Applied Physics 111, 061301 (2012); https://doi.org/10.1063/1.3688435

Enhancement of $(B H)_{\max }$ in a hard-soft-ferrite nanocomposite using exchange spring mechanism

Journal of Applied Physics 106, 073902 (2009); https://doi.org/10.1063/1.3213341

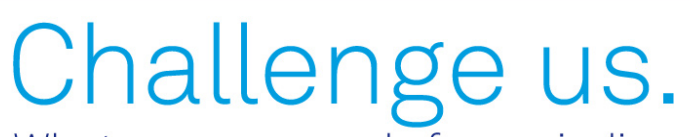
What are your needs for periodic signal detection?

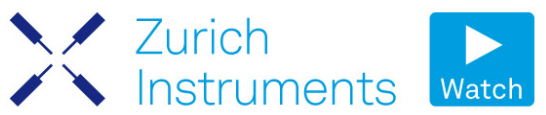

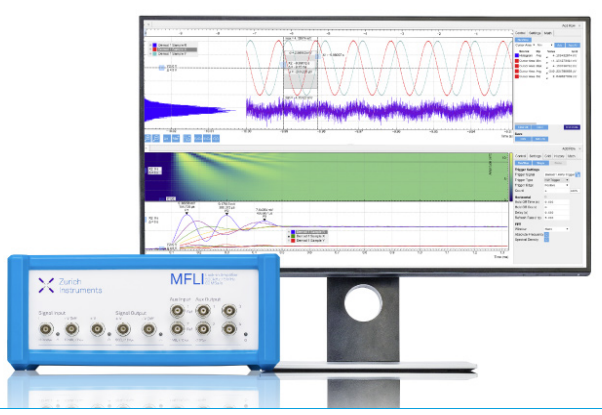

116, 154306 


\title{
Microwave characterization of magnetically hard and soft ferrite nanoparticles in K-band
}

\author{
C. Della Pina, ${ }^{1}$ E. Falletta, ${ }^{1}$ A. M. Ferretti, ${ }^{2}$ A. Ponti, ${ }^{2}$ G. G. Gentili, ${ }^{3, a)}$ V. Verri, ${ }^{3}$ \\ and R. Nesti ${ }^{4}$ \\ ${ }^{1}$ Dipartimento di Chimica, Universitá degli Studi di Milano, unità ISTM-CNR, via Golgi 19, Milano, Italy \\ ${ }^{2}$ Laboratorio di Nanotecnologie, Istituto di Scienze e Tecnologie Molecolari, Consiglio Nazionale delle \\ Ricerche, via G. Fantoli 16/15, 20138, Milano, Italy \\ ${ }^{3}$ Dipartimento di Elettronica, Informazione e Bioingegneria, Politecnico di Milano, Via Ponzio 34/5, Milano, Italy \\ ${ }^{4}$ Istituto Nazionale di Astrofisica, Largo Fermi 5, Firenze, Italy
}

(Received 1 August 2014; accepted 19 September 2014; published online 20 October 2014)

\begin{abstract}
Nano-sized magnetic particles show great promise in improving the performance of microwave absorbers with respect to the corresponding bulk materials. In this paper, magnetically hard and soft ferrite nanoparticles $\left(\mathrm{CoFe}_{2} \mathrm{O}_{4}\right.$ and $\left.\mathrm{Fe}_{3} \mathrm{O}_{4}\right)$ having an average size of 14 and $11 \mathrm{~nm}$ were prepared by co-precipitation method and characterized in terms of morphology, structure, and magnetic properties. Their permeability and permittivity were measured by a waveguide technique, embedding each sample in a host medium. Their parameters at microwave frequencies were retrieved by comparing different effective medium equations. ( 2014 AIP Publishing LLC.
\end{abstract}

[http://dx.doi.org/10.1063/1.4898138]

\section{INTRODUCTION}

Nanostructured and nanocomposite materials have emerged as alternatives to pure metals in the realization of electromagnetic shields, ${ }^{1}$ sensing circuits or antennas on non-conventional substrates ${ }^{2,3}$ with applications ranging from solar cells to radio frequency (RF) sensors and microwave absorbing coatings. ${ }^{4} \mathrm{~A}$ further attractive feature of these materials is the possibility to tune their permittivity and permeability, opening the way to materials with improved characteristics, useful for specific applications (e.g., smaller size devices or impedance matched absorbers). Different kinds of materials have been developed, effective for narrow- or broadband absorption and for low or highfrequency applications. In this regard, magnetic and dielectric materials are the most employed. Even though foams ${ }^{5}$ and polymers ${ }^{6}$ are particularly appealing for their homogeneity, lightness, and mechanical properties, magnetic composites show many advantages. ${ }^{7,8}$ It was demonstrated ${ }^{9,10}$ that nano-sized magnetic particles exhibit properties different from those of the corresponding bulk materials. Moreover, their size strongly affects their properties and it can be properly designed choosing the method of preparation. ${ }^{11-13}$ In this context, Brosseau clearly demonstrated the role of size and frequency dependence of the complex permittivity and permeability of nano- and microsized magnetic particles dispersed in a nonmagnetic metal oxide matrix. ${ }^{14}$

Recently, the microwave absorbing properties of many particulate composites (carbon black-filled polymers, $\mathrm{ZnO} /$ $\gamma-\mathrm{Fe}_{2} \mathrm{O}_{3}, \mathrm{BaTiO}_{3} / \mathrm{Ni}, \mathrm{Ni} / \gamma-\mathrm{Fe}_{2} \mathrm{O}_{3}$, etc.) have been extensively investigated, shedding light on the correlation among structure, composition, size, and electromagnetic properties. ${ }^{14-20}$ Thanks to their high magnetic loss, which contributes

\footnotetext{
a)gentili@elet.polimi.it
}

positively to microwaves absorption, magnetically soft ferrites are generally preferred to magnetically hard ones. ${ }^{21}$ However, in the GHz range the magnetic loss of soft ferrites falls down drastically, differently from some hard ferrites thus making these latter suitable as microwave absorbers. ${ }^{22,23}$ For this reason, new systems based on composite powders including hard and soft ferrites have been developed to improve the electromagnetic absorption properties of magnetic materials. ${ }^{24-27}$ Nevertheless, to the best of our knowledge, accurate assessment and comparison of the microwave absorption properties of magnetically soft- and hard-ferrites having very similar size and prepared by the same method are lacking in the scientific literature. Therefore, in this paper, we report in detail the microwave absorbing properties in K-band of magnetically soft $\left(\mathrm{Fe}_{3} \mathrm{O}_{4}\right)$ and -hard $\left(\mathrm{CoFe}_{2} \mathrm{O}_{4}\right)$ spinel ferrites of about 11 and $14 \mathrm{~nm}$ size prepared following an easy and cheap method (co-precipitation) and characterized in terms of structure and morphology.

An accurate characterization of the macroscopic electrical and magnetic parameters must necessarily rely on different techniques, according to the specific frequency bandwidths. At frequencies above $1 \mathrm{GHz}$, the waveguide characterization can be used (up to about $100 \mathrm{GHz}$ ), and in this paper, we focus our attention on the K-band $(18-26 \mathrm{GHz}$ according to IEEE designation) with a rectangular waveguide measurement system (WR42 waveguide). There are several advantages in the waveguide characterization (when applicable): a good accuracy can be obtained with rather little effort, the measurements setup does not need very large samples (the total size being comparable to the waveguide housing), and finally, the availability of both the measured reflection and transmission coefficients allows the characterization of both electric and magnetic parameters in one shot. A feature of the characterization described in this work is the mixing of the ferrites with a host medium. This has a 
two-fold effect: it allows to decrease the amount of ferrite needed to carry out the characterization, and it yields a robust solid sample that can be easily cut into bricks.

The paper is organized as follows. Materials preparation and characterization are reported in detail in Sec. II. The samples have been obtained as solids and in order to carry out the RF characterization they have been embedded in a matrix medium (polyvinyl alcohol, PVA), as reported in Sec. II B 1. In Sec. III, we present the K-band measurement setup; and in Sec. IV, we discuss the macroscopic model of the materials, including the effective medium equations of the mixture (Sec. IV A). Finally, all the results are presented and discussed in the Secs. V A and V B.

\section{MATERIALS PREPARATION AND CHARACTERIZATION}

In this section, we describe the preparation and characterization of the materials that were subsequently tested. All chemicals were purchased by Sigma Aldrich and used as received without further purification.

\section{A. Material preparation}

$\mathrm{Fe}_{3} \mathrm{O}_{4}$ and $\mathrm{CoFe}_{2} \mathrm{O}_{4}$ nanoparticles (NPs) were prepared by a co-precipitation method, in agreement with the literature. ${ }^{28,29}$

\section{Synthesis of $\mathrm{Fe}_{3} \mathrm{O}_{4} \mathrm{NPs}$}

$2.5 \mathrm{~g}$ of $\mathrm{FeSO}_{4} \cdot 7 \mathrm{H}_{2} \mathrm{O}$ and $40 \mathrm{mg}$ of PEG (PolyEthylene Glycol) were dissolved in $50 \mathrm{ml}$ of water under nitrogen atmosphere. $0.46 \mathrm{ml}$ of $\mathrm{H}_{2} \mathrm{O}_{2} 30 \%$ w/w $\left(\mathrm{Fe} / \mathrm{H}_{2} \mathrm{O}_{2}=2\right.$, molar ratio) and then an aqueous solution of $\mathrm{NaOH} 8 \mathrm{M}$ were quickly added until a $\mathrm{pH}$ value of 13 . The reaction mixture was stirred at $50^{\circ} \mathrm{C}$ for $6 \mathrm{~h}$. Finally, a black product was magnetically decanted, washed several time with water and dried in an oven at $110^{\circ} \mathrm{C}$ overnight. The resulting product was obtained with a $72 \%$ yield.

\section{Synthesis of $\mathrm{CoFe}_{2} \mathrm{O}_{4} \mathrm{NPs}$}

$145 \mathrm{mg}$ of $\mathrm{FeCl}_{3} \cdot 6 \mathrm{H}_{2} \mathrm{O}$ and $60.5 \mathrm{mg}$ of $\mathrm{CoCl}_{2} \cdot 6 \mathrm{H}_{2} \mathrm{O}$ $(\mathrm{Fe} / \mathrm{Co}=2$, atomic ratio) were dissolved in $100 \mathrm{ml}$ of deionized water and stirred for $20 \mathrm{~min}$ at $80^{\circ} \mathrm{C}$ under nitrogen atmosphere. Then, an aqueous solution of $\mathrm{NaOH} 8 \mathrm{M}$ was quickly added until a $\mathrm{pH}$ value of 13 and the solution was vigorously stirred for $2 \mathrm{~h}$. A dark product was recovered by magnetic separation, washed with distilled water until neutral $\mathrm{pH}$ and dried at $110^{\circ} \mathrm{C}$ in an oven overnight. The resulting product was obtained with a $91 \%$ yield.

\section{B. Materials characterization}

$\mathrm{X}$-ray powder diffraction (XRPD) analyses were carried out using a Rigaku D IIIMAX horizontal-scan powder diffractometer with $\mathrm{Cu} \mathrm{K}_{\alpha}$ radiation. The $\mathrm{Fe} / \mathrm{Co}$ atomic ratio in spinel ferrite was determined by atomic absorption spectroscopy (AAS) on an AAnalyst 100 PerkinElmer instrument. The products were morphologically characterized by transmission electron microscopy (TEM) using a Zeiss
LIBRA-200FE microscope. The measurement of the magnetic properties was carried out by a Quantum Design MPMS XL-5 SQUID magnetometer.

\section{Specimen preparation for RF measurements}

The specimens for microwave absorption measurements were prepared as rectangular bricks using a home-made mold. Each sample was embedded in a host medium (PVA) by mixing $1 \mathrm{~g}$ of nanoparticles with $3 \mathrm{~g}$ of commercial PVA (dispersion of the material in water ca. 50\%). The resulting viscous fluid was deposited in the mold layer by layer. Before depositing the next layer, each one was left standing for $20 \mathrm{~min}$ to ensure complete water evaporation. When the specimen reached the desired thickness $(0.2 \mathrm{~cm})$, it was removed from the mold, dried in an oven at $60^{\circ} \mathrm{C}$ for $24 \mathrm{~h}$, and polished with sandpaper. PVA has been selected as a host medium since, after mixing with the material and evaporation of the water content, it can be easily and rather accurately cut in brick form without breaking the sample.

\section{K-BAND MEASUREMENT SETUP}

The RF measurement setup comprises a vector network analyzer and a rectangular waveguide calibration kit. We used WR42 waveguide $(a=10.668 \mathrm{~mm}, \quad b=4.318 \mathrm{~mm})$, covering the whole K-band $(18-26 \mathrm{GHz})$. After calibration in the frequency domain, the network analyzer measures $S_{11}$, $S_{12}, S_{21}$, and $S_{22}$ parameters in magnitude and phase. The device under test (DUT) is a rectangular brick entirely filling the waveguide transverse cross-section and having length $L$ (see Fig. 1). The brick is a solid material that can be easily cut to the desired size. The composites included in the DUT must represent a convenient volume fraction of the sample, neither too small (leading to reduced accuracy in the reconstruction of the electric and magnetic parameters) nor too large (because of fragility issues in the DUT). From the measured scattering matrix, the electric and magnetic parameters of the composites can be obtained by inversion of the effective medium equations. This is described in Sec. IV.

\section{MATERIALS MODELING IN K-BAND}

The DUT is a rectangular waveguide section of length $L$ entirely filled with a homogeneous composite material. The simple model used for the materials is the following: an admittivity $\sigma+j \omega \epsilon_{0}\left(\epsilon_{r}^{\prime}-j \epsilon_{r}^{\prime \prime}\right)$ for the electric part and a relative magnetic permeability $\mu_{r}^{\prime}-j \mu_{r}^{\prime \prime}$ for the magnetic part, where $\omega$ is the angular frequency, $\epsilon_{0}=8.854 \cdot 10^{-12} \mathrm{~F} / \mathrm{m}$, and a time dependence $e^{j \omega t}$ is assumed and suppressed.

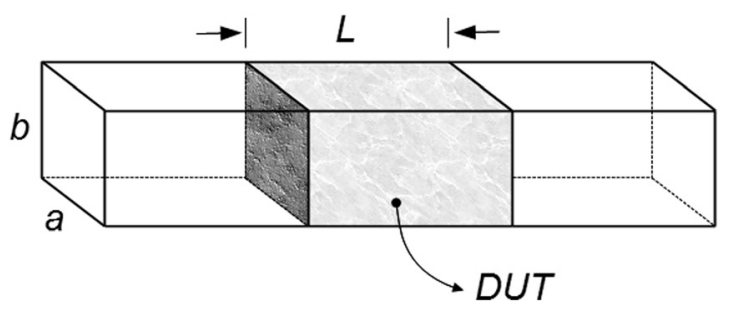

FIG. 1. Waveguide partially filled with sample under test. 
The parameter $\sigma$ is a frequency independent conductivity $(\mathrm{S} / \mathrm{m}), \epsilon_{r}^{\prime \prime}$ represents dielectric type losses (the equivalent conductivity linearly increasing with frequency) and $\mu_{r}^{\prime \prime}$ represents magnetic type losses. Finally, the frequency independent $\epsilon_{r}^{\prime}$ and $\mu_{r}^{\prime \prime}$ are the usual relative permittivity and permeability. Since ohmic losses of the hosting waveguide walls have been characterized and de-embedded from the DUT measurement, this model is capable to represent gentle variations of material losses with frequency, and it was found adequate to represent the materials in the whole K-band. After measuring the scattering parameters, one can find the admittivity and permeability of the composite materials by introducing at least squares error between the measured and computed scattering parameters and by minimizing the error over the whole frequency band by a gradient search. The scattering parameters of a rectangular waveguide section of length $L$, filled with an unknown medium, can be expressed as follows: using the pedix " $e$ " to refer to the composite medium and " 0 " to refer to the empty waveguide, the analytically computed scattering parameters are

$$
\begin{aligned}
& S_{11}=S_{22}=\frac{\left(Z_{e}^{2}-Z_{0}^{2}\right) \tanh \gamma_{e} L}{2 Z_{e} Z_{0}+\left(Z_{e}^{2}+Z_{0}^{2}\right) \tanh \gamma_{e} L}, \\
& S_{21}=S_{12}=\left(1+S_{11}\right) \frac{1+\Gamma}{1+\Gamma e^{-2 \gamma_{e}} L} e^{-\gamma_{e} L},
\end{aligned}
$$

where $Z_{0}=1 / \gamma_{0}, Z_{e}=\mu_{r e} / \gamma_{e}$

$$
\begin{gathered}
\gamma_{0}=\sqrt{(\pi / a)^{2}-\omega^{2} \mu_{0} \epsilon_{0}}, \\
\gamma_{e}=\sqrt{(\pi / a)^{2}+j \omega \mu_{e}\left(\sigma_{e}+j \omega \epsilon_{e}\right)}
\end{gathered}
$$

and finally $\Gamma=\left(Z_{0}-Z_{e}\right) /\left(Z_{0}+Z_{e}\right)$ (see, e.g., Ref. 30 and references therein).

In the process, it was observed that the use of a frequency dependent admittivity was important. As an output of the measurement and characterization process, one gets the admittivity and permeability of the composite material. From the latter, the parameters of the nanoinclusions can be obtained by inverting the effective medium equation.

\section{A. Effective medium equations}

An effective medium is a medium obtained by mixing two or more different species into a single mixture. There are several models that can be used to represent the electric and magnetic parameters of a mixture, and there are a large amount of literature in this regard. ${ }^{31-34}$ All of them consider the effective medium as a set of small inclusions of different shapes into a matrix (host medium). In theory, in order to be able to neglect mutual interactions among the inclusions, these latter should be "far enough," i.e. the volume fraction should be small. This is not always the case for the materials analyzed in this work, and moreover, the actual shapes of the inclusions are unknown. For these reasons, in order to reduce the uncertainty in the evaluation of the nanoinclusions parameters, we considered two different rules for the effective medium: the Maxwell Garnett rule (MG) and the Bruggeman rule (BR). Both mixing rules used in this paper assume small spherical inclusions, but using both rules allows a cross check of the results obtained. MG model is known to be more accurate at small volume fractions, and BR model has the property of being symmetrical in the effective medium formulas and is more suitable at larger volume fractions. ${ }^{35}$ As most mixing rules, they have been derived in a quasistatic regime and assume no particle agglomerations in the mixture. Although for low contrast the Looyenga mixing rule is preferable, ${ }^{36}$ in our case it did not show significant differences with respect to the BR model. Using formulas for two phase mixtures and using pedix "eff" to refer to the parameters of the effective medium, " $h$ " for the host medium, and " $x$ " for the inclusions, one finds for MG theory

$$
\psi_{\mathrm{eff}}=\psi_{h}+3 f_{v} \psi_{h} \frac{\psi_{x}-\psi_{h}}{\psi_{x}+2 \psi_{h}-f_{v}\left(\psi_{x}-\psi_{h}\right)}
$$

whereas for BR

$$
\frac{3 f_{v} \psi_{\mathrm{eff}}}{2 \psi_{\mathrm{eff}}+\psi_{x}}+\frac{3\left(1-f_{v}\right) \psi_{\mathrm{eff}}}{2 \psi_{\mathrm{eff}}+\psi_{h}}=1,
$$

where $f_{v}$ is the volume fraction of the inclusions and $\psi$ is the admittivity or permeability of the various materials.

From the measured data, the parameter $\psi_{\text {eff }}$ has been obtained and by the same measurement technique the parameters of the host medium can be obtained, so that by inverting Eqs. (5) and (6) one finds

$$
\psi_{x}=\psi_{h} \frac{2 \psi_{h}\left(1-f_{v}\right)-\psi_{\mathrm{eff}}\left(2+f_{v}\right)}{\psi_{\mathrm{eff}}\left(1-f_{v}\right)-\psi_{h}\left(1+2 f_{v}\right)}
$$

for MG and

$$
\psi_{x}=\psi_{\text {eff }} \frac{\psi_{h}\left(2-3 f_{v}\right)-2 \psi_{\text {eff }}}{\psi_{\text {eff }}\left(1-3 f_{v}\right)-\psi_{h}}
$$

for BR.

Note that even from the simple model of effective medium used in this work, a rather complex frequency dependence for the electric part can appear as a result of effective medium equations. In our case, however, the nanoinclusions have been observed to be accurately represented by a complex constant relative permittivity $\epsilon_{r x}^{\prime}-j \epsilon_{r x}^{\prime \prime}$ and a relative permeability $\mu_{r x}^{\prime}-j \mu_{r x}^{\prime \prime}$. For this reason, the final results for the nanoparticles are shown in tabular form.

\section{RESULTS}

In this section, spectroscopic (Subsection A) and RF characterization results (Subsection B) are reported.

\section{A. Magnetic nanoparticles characterization}

At first, the $\mathrm{Fe} / \mathrm{Co}$ atomic ratio was determined by AAS for two different $\mathrm{CoFe}_{2} \mathrm{O}_{4}$ NPs batches and the results are reported in Table I.

As shown in the Table, the experimental results are in agreement with the stoichiometric ratio $(\mathrm{Fe} / \mathrm{Co}=2)$. The diffractograms (not reported) show the characteristic Bragg 
TABLE I. AAS analyses of Fe and Co of two $\mathrm{CoFe}_{2} \mathrm{O}_{4}$ NPs batches.

\begin{tabular}{lc}
\hline \hline Sample & Fe/Co (atomic ratio) \\
\hline 1 & 1.97 \\
2 & 2.10 \\
\hline \hline
\end{tabular}

diffraction peaks corresponding to the Miller indices for the reflection planes (220), (311), (400), (422), (511), (440) at $2 \theta=30.3^{\circ}, 35.6^{\circ}, 43.2^{\circ}, 53.6^{\circ}, 57.1^{\circ}$ and $62.8^{\circ}$, respectively, for cubic ferrites with spinel structure. ${ }^{37,38}$

The mean and the standard deviation of the nanoparticle diameter distribution were evaluated by analyzing the diffractograms by the Rietveld method as implemented in the MAUD program. ${ }^{39,40}$ The size distribution of the nanoparticles was modeled as a Normal (Gaussian) distribution, which gave better fit than the Log-Normal distribution. The best-fit parameters are reported in Table II. The mean NPs diameter obtained by XRD analyses are consistent with the TEM characterization (Fig. 2). From TEM images, it can be appreciated that the ferrite NPs form large agglomerates and seem to be in direct contact to each other. A detailed morphological description of such aggregated nanoparticles is very difficult. Manual measurement of the TEM images allowed us to estimate that the nanoparticle diameter ranges from 3 to $25 \mathrm{~nm}$, in agreement with XRD results. The aggregates range in size from several hundred nanometers to several micrometers and are often thick enough to be opaque to the electron beam. The smaller NPs are spheroidal, while the larger ones have polyhedral shape.

The magnetization $M$ of the ferrite NPs was measured as a function of applied field at $5 \mathrm{~K}$. The thermal behavior of $\mathrm{M}$ was measured in field-cooling (FC) and zero-field-cooling (ZFC) modes in the range $300-5 \mathrm{~K}$. The magnetization curves are shown in Fig. 3 and the main magnetic parameters are collected in Table III.

The dramatic difference in the magnetic behaviour of the two NPs types is apparent from these results. The magnetite NPs sample is magnetically very soft, with vanishing remanence $M_{r}$ and coercivity $H_{c}$. The cobalt ferrite NPs instead show significant $M_{r}$ and very high $H_{c}$. These largely different coercivity values arise from the direct contact between the NPs which provide a pathway for electronic
TABLE II. Size distribution parameters of $\mathrm{Fe}_{3} \mathrm{O}_{4}$ and $\mathrm{CoFe}_{2} \mathrm{O}_{4}$ nanoparticles.

\begin{tabular}{lcc}
\hline \hline Sample & Mean diameter $(\mathrm{nm})$ & Diameter standard deviation (nm) \\
\hline $\mathrm{Fe}_{3} \mathrm{O}_{4}$ & $11 \pm 1$ & $0.42 \pm 0.09$ \\
$\mathrm{CoFe}_{2} \mathrm{O}_{4}$ & $14 \pm 1$ & $0.62 \pm 0.02$ \\
\hline \hline
\end{tabular}

exchange coupling between NPs. In this case, the coercivity reduction from the isolated NPs value can be expressed as ${ }^{41}$

$$
H_{c}=H_{a n}\left[\frac{D}{\delta}\right]^{6},
$$

where $H_{a n}$ is the anisotropy field, $D$ is the NPs size, and $\delta$ is the Bloch wall width. For soft $\mathrm{Fe}_{3} \mathrm{O}_{4}$, Eq. (9) gives $H_{c}<1$ Oe. When the measured $H_{c}$ for cobalt ferrite is inserted in Eq. (9), one obtains $D \simeq 16 \mathrm{~nm}$, in good agreement with XRD and TEM data.

The thermal behaviour of the magnetization of the two NPs types is also very dissimilar (see Figs. 3(d)-3(f)). The behaviour of the $\mathrm{Fe}_{3} \mathrm{O}_{4}$ NPs is clear evidence of the presence of blocking-unblocking superparamagnetic (SPM) processes with a distribution of the energy barriers extending from 5 to $300 \mathrm{~K}$ and peaking at $80 \mathrm{~K}$ (Fig. 3(e)). Such distribution, broader than that usually observed for isolated NPs, gives an idea of the many different interparticle interactions present in the NP sample. The temperature dependence of the magnetization of $\mathrm{CoFe}_{2} \mathrm{O}_{4}$ NPs (Fig. 3(f)) follows a Bloch $T^{3 / 2}$ law with parameters very close to those of the bulk material. This behaviour confirms the importance of the interparticle exchange coupling which extends the energy barrier for magnetization reversal towards larger values, making the cobalt ferrite NPs behave as the bulk material.

\section{B. K-band characterization}

The results of K-band characterization have been organized as follows. First, the measured data are presented, in order to verify the agreement between measured S-parameters and those obtained by the model of the effective medium (computed analytically). Then, the results for the materials are shown in tabular form. Finally, the results are discussed and commented.

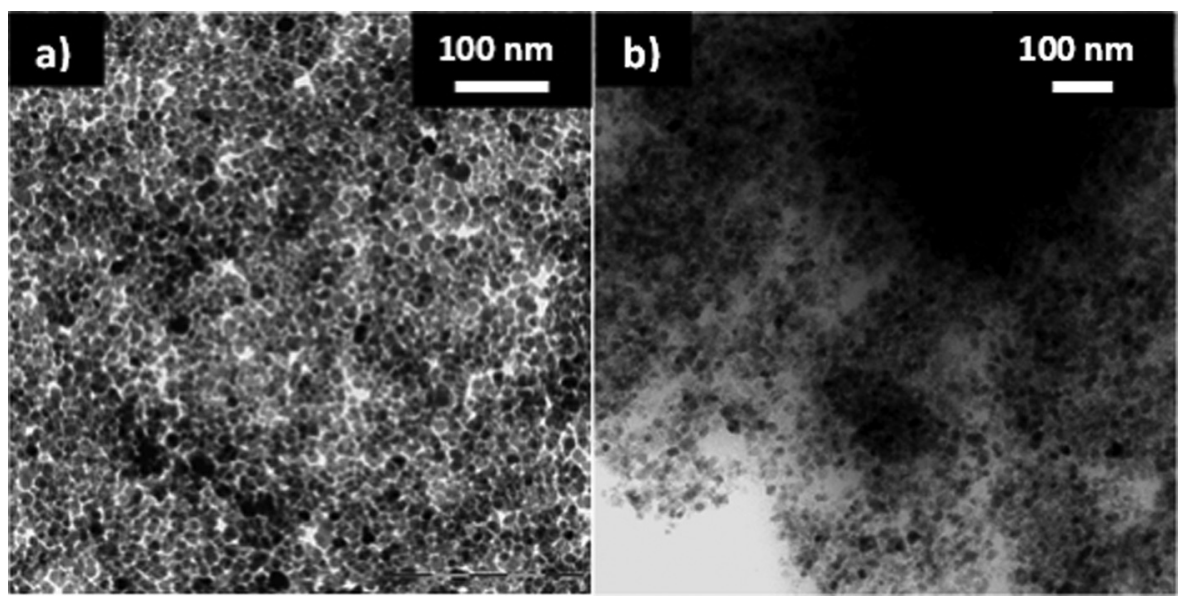

FIG. 2. TEM images of (a) $\mathrm{Fe}_{3} \mathrm{O}_{4}$ and (b) $\mathrm{CoFe}_{2} \mathrm{O}_{4}$ NPs. 
a)

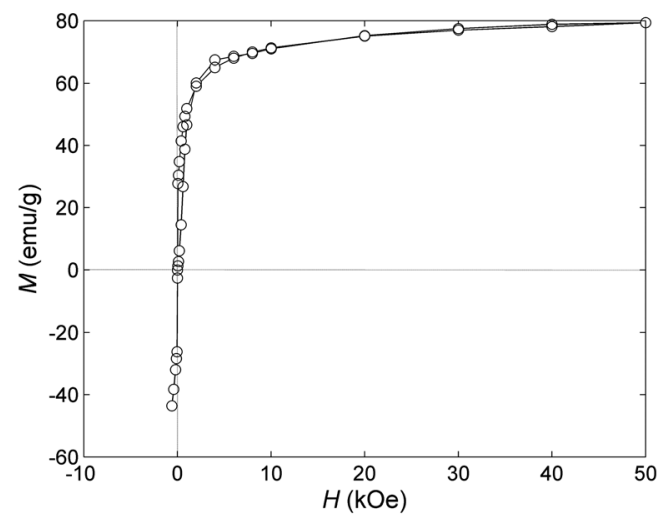

c)

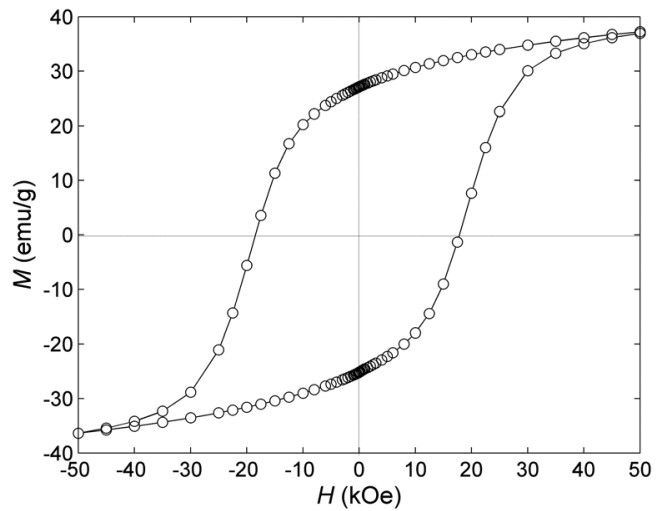

e)

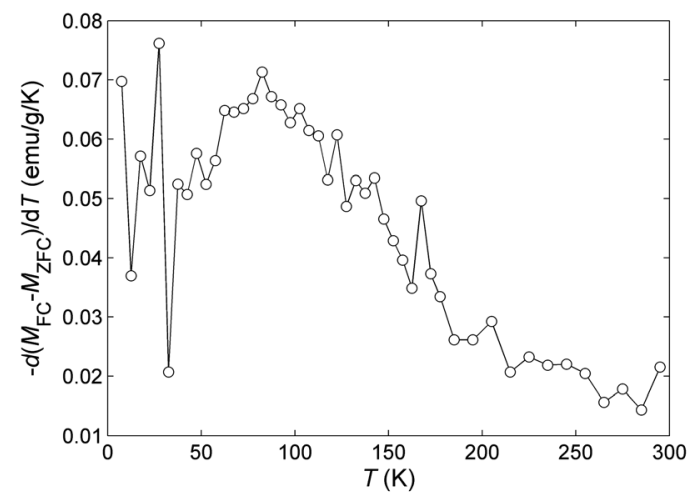

b)

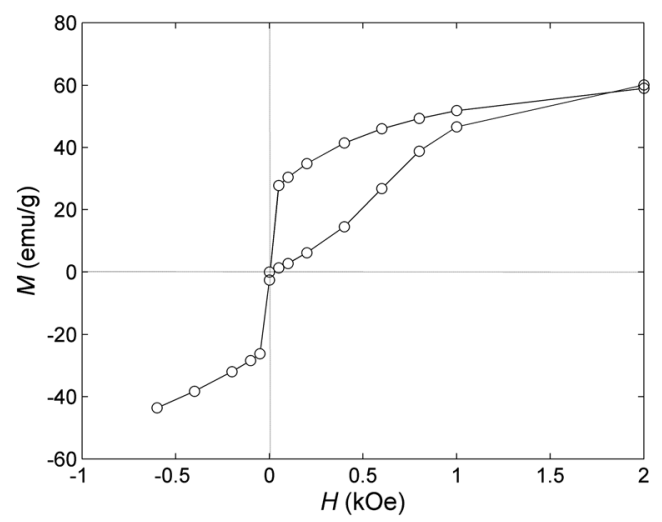

d)

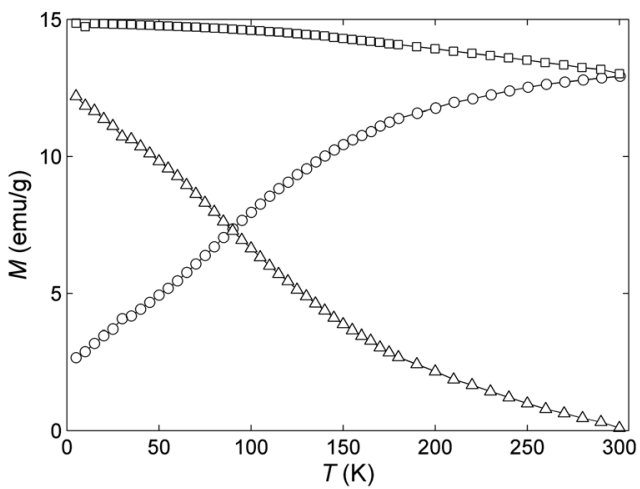

f)

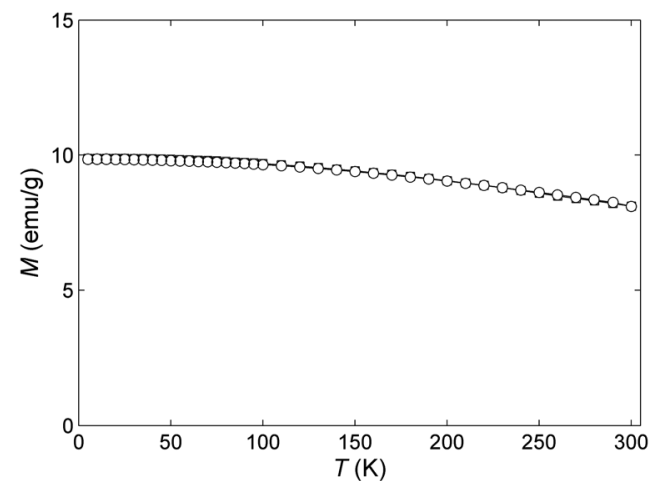

FIG. 3. Magnetization of ferrite nanoparticles. (a) and (b) Magnetization/demagnetization curve of $\mathrm{Fe}_{3} \mathrm{O}_{4} \mathrm{NPs}$ at $5 \mathrm{~K}$. (c) Field-cooled magnetization isotherm of $\mathrm{CoFe}_{2} \mathrm{O}_{4} \mathrm{NPs}$ at $5 \mathrm{~K}$. (d) FC (squares) and ZFC (circles) magnetization of $\mathrm{Fe}_{3} \mathrm{O}_{4}$ NPs along with their difference $M_{F C}-M_{Z F C}$ (triangles). (e) Derivative $-d\left(M_{F C}-M_{Z F C}\right) / d T$ of $\mathrm{Fe}_{3} \mathrm{O}_{4}$ NPs. (f) FC (squares) and $\mathrm{ZFC}$ (circles) magnetization of $\mathrm{CoFe}_{2} \mathrm{O}_{4} \mathrm{NPs}$.

At first, a comparison between measured S-parameters of PVA and PVA model is presented in Fig. 4. Although the agreement is quite good, it is not perfect and this can be due either to

TABLE III. Magnetic properties of ferrite nanoparticles at $5 \mathrm{~K}$.

\begin{tabular}{lcr}
\hline \hline & $\mathrm{Fe}_{3} \mathrm{O}_{4}$ & $\mathrm{CoFe}_{2} \mathrm{O}_{4}$ \\
\hline$M_{50}(\mathrm{emu} / \mathrm{g})^{\mathrm{a}}$ & 79 & 37 \\
$M_{r}(\mathrm{emu} / \mathrm{g})$ & 0 & 27 \\
$H_{c}(\mathrm{Oe})$ & 0 & 18500 \\
\hline \hline
\end{tabular}

${ }^{\mathrm{a}} M_{50}$ is the magnetization at $H=+50 \mathrm{kOe}$. imperfect cutting of the brick sample or to some inhomogeneities that appear in the brick after water evaporation (small air bubbles). However, since the disagreement is confined to a small range of frequencies around $24 \mathrm{GHz}$, the impact on the retrieved parameters of the unknown medium is minimum, since the inversion of the effective medium equations is carried out in the frequency domain. Note also that measured parameters $\left|S_{11}\right|$ and $\left|S_{22}\right|$ are not equal, which is a further indication of some small asymmetry in the sample realization (cutting or mixing). The frequency independent parameters obtained for PVA are $\sigma=0$, $\epsilon_{r}^{\prime}=2.68, \epsilon_{r}^{\prime \prime}=0.07, \mu_{r}^{\prime}=1.07$, and $\mu_{r}^{\prime \prime}=0.03$. These have been used as host parameters $\psi_{h}$ in the further processing. 
(a) PVA: S11, S22 (dB)

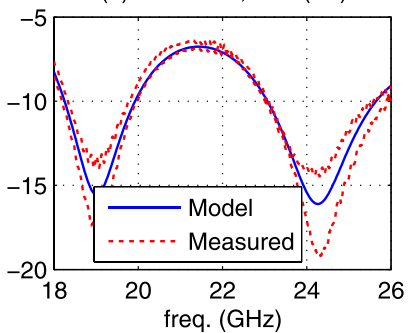

(c) PVA: Phase S11 (deg)

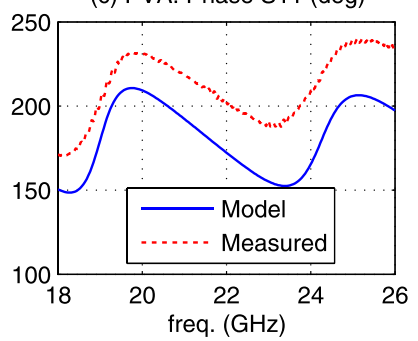

(b) PVA: S21 (dB)

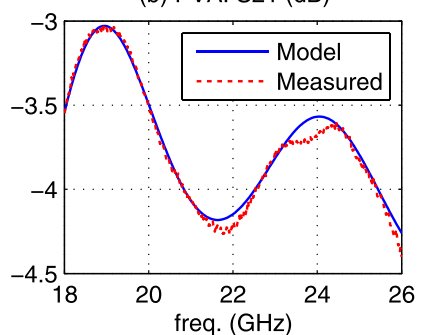

(d) PVA: Phase S21 (deg)

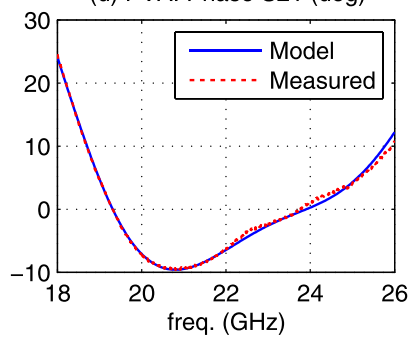

FIG. 4. Comparison between model and measurements for the host medium (PVA).

The measured results for $\mathrm{CoFe}_{2} \mathrm{O}_{4}$ and the comparison with model are shown in Fig. 5. Also in this case there is a difference in the measured S-parameters $\left|S_{11}\right|$ and $\left|S_{22}\right|$, but the agreement is very good. Finally, the results for $\mathrm{Fe}_{3} \mathrm{O}_{4}$ are shown in Fig. 6. Good results have been obtained in this case too. Note that in all the three Figures 4-6 a linear term with frequency has been subtracted from the phase of $S_{21}$ (modeled and measured) in order to make a sharper comparison.

From the measured parameters of the host and the effective medium, one can derive the parameters of the nanoparticles by using (7) and (8). The results are shown in Tables IV and V. It was observed that in the whole K-band the nanoparticles are rather accurately represented by a frequency independent complex relative permittivity $\left(\epsilon_{r}^{\prime}-j \epsilon_{r}^{\prime \prime}\right)$ and relative permeability $\left(\mu_{r}^{\prime}-j \mu_{r}^{\prime \prime}\right)$. As shown in Tables IV and V, there is a good agreement between MG and BR models and

(a) CoFe2O4: S11, S22 (dB)

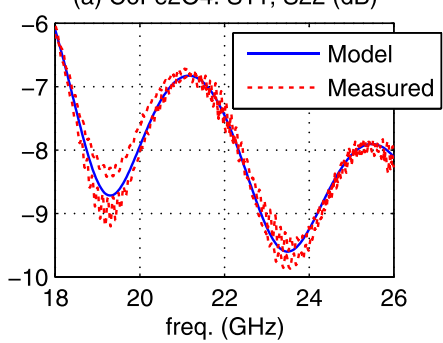

(c) CoFe2O4: Phase $\mathrm{S} 11$ (deg)

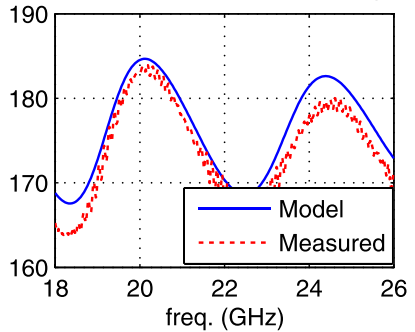

(b) CoFe2O4: S21 (dB)

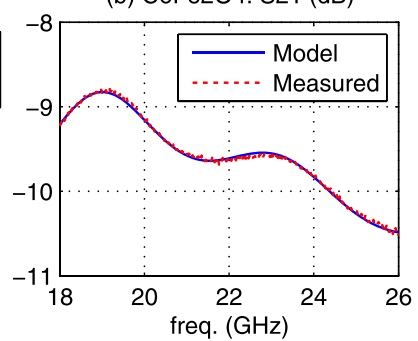

(d) CoFe2O4: Phase S21 (deg)

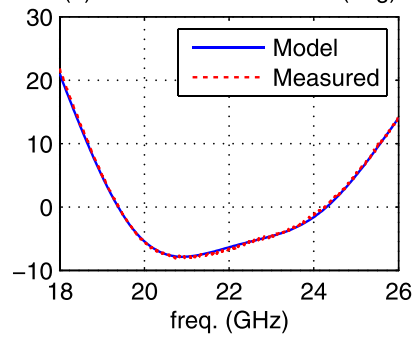

FIG. 5. Comparison between model and measurements for mixture of PVA and $\mathrm{CoFe}_{2} \mathrm{O}_{4}\left(f_{v}=0.6\right)$.

(a) Fe3O4: S11, S22 (dB)

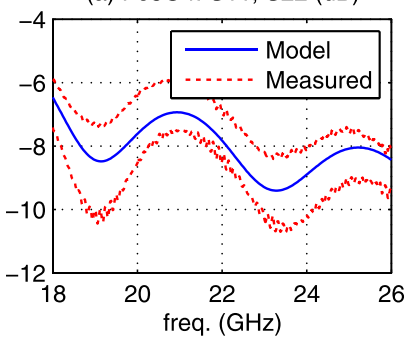

(c) Fe3O4: Phase S11 (deg)

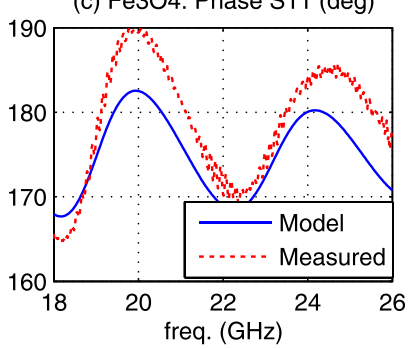

(b) Fe3O4: S21 (dB)

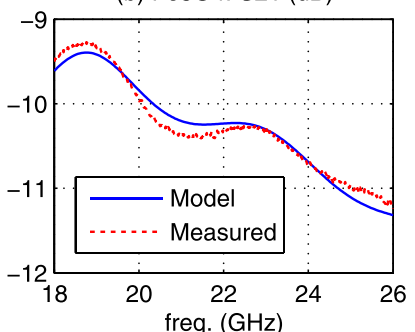

(d) Fe3O4: Phase S21 (deg)

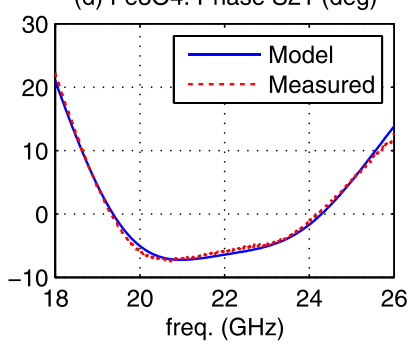

FIG. 6. Comparison between model and measurements for mixture of PVA and $\mathrm{Fe}_{3} \mathrm{O}_{4}\left(f_{v}=0.61\right)$.

TABLE IV. Derived data for the nanoparticles (electric part). MG and BR models.

\begin{tabular}{llcc}
\hline \hline & $f_{v}$ & $\epsilon_{r}^{\prime}(\mathrm{MG}-\mathrm{BR})$ & $\epsilon_{r}^{\prime \prime}(\mathrm{MG}-\mathrm{BR})$ \\
\hline $\mathrm{PVA}$ & 1 & $2.68-2.68$ & $0.07-0.07$ \\
$\mathrm{CoFe}_{2} \mathrm{O}_{4}$ & 0.6 & $4.52-4.49$ & $0.76-0.73$ \\
$\mathrm{Fe}_{3} \mathrm{O}_{4}$ & 0.61 & $4.45-4.43$ & $0.90-0.87$ \\
\hline \hline
\end{tabular}

TABLE V. Derived data for the nanoparticles (magnetic part). MG and BR models.

\begin{tabular}{llcc}
\hline \hline & $f_{v}$ & $\mu_{r}^{\prime}(\mathrm{MG}-\mathrm{BR})$ & $\mu_{r}^{\prime \prime}(\mathrm{MG}-\mathrm{BR})$ \\
\hline $\mathrm{PVA}$ & 1 & $1.07-1.07$ & $0.03-0.03$ \\
$\mathrm{CoFe}_{2} \mathrm{O}_{4}$ & 0.6 & $1.09-1.09$ & $0.0-0.0$ \\
$\mathrm{Fe}_{3} \mathrm{O}_{4}$ & 0.61 & $1.11-1.11$ & $0.2-0.2$ \\
\hline \hline
\end{tabular}

this provides some more confidence in the results obtained. Apparently, this results are in partial disagreement with, ${ }^{34}$ but it must be said that the frequency range in our case is higher and smaller in relative extent.

\section{CONCLUSIONS}

Magnetically-hard and -soft nanoparticles with about $14 \mathrm{~nm}\left(\mathrm{CoFe}_{2} \mathrm{O}_{4}\right)$ and $11 \mathrm{~nm}\left(\mathrm{Fe}_{3} \mathrm{O}_{4}\right)$ mean diameter were prepared and characterized by AAS, X-ray diffractometry, and TEM. Given the measured relative permeability of PVA (different from 1 due to imperfections in the sample realization), the small $\mu^{\prime \prime}$ of $\mathrm{Fe}_{3} \mathrm{O}_{4}$ NPs and the vanishing for $\mathrm{CoFe}_{2} \mathrm{O}_{4} \mathrm{NPs}$ can be understood by recalling that the initial static permeability of soft $\mathrm{Fe}_{3} \mathrm{O}_{4}$ NPs is much larger than that of hard $\mathrm{CoFe}_{2} \mathrm{O}_{4}{ }^{42}$ This difference most probably is retained in the investigated microwave range. However, we observed that $\mathrm{Fe}_{3} \mathrm{O}_{4}$ NPs are mostly (though not completely) in the superparamagnetic regime at RT, whereas $\mathrm{CoFe}_{2} \mathrm{O}_{4}$ nanoparticles are still in blocked state at RT due to the strong 
interparticle exchange interaction. This suggests that there can be another dynamic mechanism which leads to an increase of $\mu^{\prime \prime}$ of $\mathrm{Fe}_{3} \mathrm{O}_{4}$ NPs with respect to $\mathrm{CoFe}_{2} \mathrm{O}_{4}$ NPs, i.e. the interplay between the intrinsic ferromagnetic resonance at $f_{\text {res }}=\gamma H_{a n} / 2 \pi$ (ca. $1-2 \mathrm{GHz}$ for cubic ferrites) and the MW absorption occurring when the MW frequency matches the magnetization inversion frequency $f_{\mathrm{SPM}}$ $=f_{0} \exp (-K V / k T)$ due to SPM relaxation. ${ }^{43,44}$ Here, $\mathrm{K}$ is the magnetocrystalline anisotropy constant and $V$ is the NPs volume, which makes $f_{\text {SPM }}$ strongly size dependent and possibly larger than $f_{\text {res }}$. The discussion is best based on the $H_{t h} /$ $H_{a n}$ ratio, where $H_{t h}=k T / V M_{\text {sat }}$ represents the thermal energy in magnetic field units $\left(M_{\text {sat }}\right.$ is the saturation magnetization). For $\mathrm{CoFe}_{2} \mathrm{O}_{4} \mathrm{NPs}$ with about $14 \mathrm{~nm}$ diameter, $H_{t h} /$ $H_{a n}<1$ and no MW absorption due to SPM is expected. Being the MW frequency much higher than the intrinsic $f_{\text {res }}$, the $\mathrm{CoFe}_{2} \mathrm{O}_{4}$ NPs are in the "no response" regime, where $\mu^{\prime \prime}=0$.

Conversely, $\mathrm{Fe}_{3} \mathrm{O}_{4}$ NPs with size less than $7 \mathrm{~nm}$ have $H_{t h} / H_{a n}>1$ and may absorb MW radiation by SPM. It has been reported that 8.8 (3) $\mathrm{nm} \mathrm{Fe}_{3} \mathrm{O}_{4} \mathrm{NPs}$ have a peak in $\mu^{\prime \prime}$ at 1.3 (5.3) $\mathrm{GHz}^{43}$ The smaller $\mathrm{Fe}_{3} \mathrm{O}_{4}$ NPs may then make a (small) contribution to $\mu^{\prime \prime}$ in $\mathrm{K}$ band, in any case larger than that of $\mathrm{CoFe}_{2} \mathrm{O}_{4} \mathrm{NPs}$, and be partially responsible for the small but non-vanishing observed $\mu^{\prime \prime}$. This different behavior between $\mathrm{Fe}_{3} \mathrm{O}_{4}$ and $\mathrm{CoFe}_{2} \mathrm{O}_{4}$ NPs can be traced back to the large difference in magnetocrystalline anisotropy (via $H_{a n}$ ), that is, to their magnetically soft and hard character.

As for the RF part, the specimens prepared for K-band measurements by mixing the nanoparticles with a matrix medium were proven a viable solution for RF testing. The process allowed to estimate with good accuracy the electric and magnetic parameters of the materials over the wide frequency bandwidth $18-26 \mathrm{GHz}$. A value for $\tan \delta_{\epsilon}=\epsilon^{\prime \prime} / \epsilon^{\prime}$ of about 0.2 has been obtained throughout the K-band.

\section{ACKNOWLEDGMENTS}

The work has been financially supported by the Cariplo Foundation through the Project 2012-0872 "Magnetic polymer nanoparticle-filled conductive polymer composites for EMI reduction." We would like to thank the anonymous reviewers for their valuable help to improve the paper.

\footnotetext{
${ }^{1}$ A. A. Al-Ghamdi, O. A. Al-Hartomy, F. Al-Solamy, A. A. Al-Ghamdi, and F. El-Tantawy, "Electromagnetic wave shielding and microwave absorbing properties of hybrid epoxy resin/foliated graphite nanocomposites," J. Appl. Polym. Sci. 127(3), 2227-2234 (2013).

${ }^{2}$ A. Rashidian, D. M. Klymyshyn, M. T. Aligodarz, M. Boerner, and J. Mohr, "Photoresist-based polymer resonator antennas: lithography fabrication, strip-fed excitation, and multimode operation," IEEE Antennas Propag. Mag. 53(4), 16-27 (2011).

${ }^{3}$ N. J. Kirsch, N. A. Vacirca, T. P. Kurzweg, A. K. Fontecchio, and K. R. Dandekar, "Performance of transparent conductive polymer antennas in a MIMO ad-hoc network," in IEEE 6th International Conference on Wireless and Mobile Computing, Networking and Communications (WiMob) (2010), Vol. 9, pp. 11-13.

${ }^{4}$ I. Kong, S. H. Ahmada, M. H. Abdullah, D. Hui, A. NazlimYusoff, and D. Puryanti, "Magnetic and microwave absorbing properties of magnetitethermoplastic natural rubber nanocomposites," J. Magn. Magn. Mater. 322, 3401-3409 (2010).

${ }^{5}$ D. R. Smith, S. Schultz, N. Kroll, M. Sigalas, K. M. Ho, and C. M. Soukoulis, Appl. Phys. Lett. 65(5), 645 (1994).
}

${ }^{6}$ H. John, R. M. Thomas, J. Jacob, K. T. Mathew, and R. Joseph, "Conducting polyaniline composites as microwave absorbers," Polym. Compos. 28(5), 588-592 (2007).

${ }^{7}$ Y. Naito and K. Suetake, "Application of ferrite to electromagnetic wave absorber and its characteristics," IEEE Trans. Microwave Theory Tech. 19, 65-72 (1971).

${ }^{8}$ W. Jing, Z. Hong, B. Shuxin, C. Ke, and Z. Changrui, "Microwave absorbing properties of rare-earth elements substituted W-type barium ferrite," J. Magn. Magn. Mater. 312, 310-313 (2007).

${ }^{9}$ S. P. Gubin, Y. A. Koksharov, G. B. Khomutov, and G. Y. Yurkov, Russ. Chem. Rev. 74(6), 489-520 (2005).

${ }^{10} \mathrm{C}$. Brosseau and P. Talbot, "Effective magnetic permeability of $\mathrm{Ni}$ and $\mathrm{Co}$ micro- and nanoparticles embedded in a $\mathrm{ZnO}$ matrix," J. Appl. Phys. 97, 104325 (2005).

${ }^{11}$ R. H. Kodama, J. Magn. Magn. Mater. 200, 359-372 (1999).

${ }^{12}$ J. L. Dormann, D. Fiorani, and E. Tronc, "Magnetic relaxation in fine particle systems," Adv. Chem. Phys. 98, 283-494 (1997).

${ }^{13}$ C. Brosseau, J. Ben Youssef, P. Talbot, and A.-M. Konn, "Electromagnetic and magnetic properties of multicomponent metal oxides heterostructures: Nanometer versus micrometer-sized particles," J. Appl. Phys. 93(11), 9243-9256 (2003).

${ }^{14} \mathrm{C}$. Brosseau, "Generalized effective medium theory and dielectric relaxation in particle-filled polymeric resins," J. Appl. Phys. 91(5), 3197-3204 (2002).

${ }^{15} \mathrm{~V}$. Castel, C. Brosseau, and J. Ben Youssef, "Magnetoelectric effect in $\mathrm{BaTiO}_{3} / \mathrm{Ni}$ particulate nanocomposites at microwave frequencies," J. Appl. Phys. 106, 064312 (2009).

${ }^{16}$ C. Brosseau, S. Mallégol, P. Quéffelec, and J. Ben Youssef, "Nonreciprocal electromagnetic properties of nanocomposites at microwave frequencies," Phys. Rev. B 70, 092401 (2004).

${ }^{17}$ J. Ben Youssef and C. Brosseau, "Magnetization damping in two-component metal oxide micropowder and nanopowder compacts by broadband ferromagnetic resonance measurements," Phys. Rev. B 74, 214413 (2006).

${ }^{18}$ S. Mallègol, C. Brosseau, P. Quéffelec, and A. M. Konn, "Measurement of the permeability tensor in nanophases of granular metal oxides and fieldinduced magnetic anisotropy," Phys. Rev. B 68, 174422 (2003).

${ }^{19} \mathrm{~V}$. Castel and C. Brosseau, "Magnetic field dependence of the effective permittivity in $\mathrm{Ba} \mathrm{Ti}_{3} \mathrm{Ni}$ nanocomposites observed via microwave spectroscopy," Appl. Phys. Lett. 92, 233110 (2008).

${ }^{20}$ J. M. Song, "Studies on microwave absorbers prepared with cast alnico magnets," J. Korean Phys. Soc. 46(4), 937-940 (2005).

${ }^{21}$ S. Sugimoto, K. Okayama, S. I. Kondo, H. Ota, M. Kimura, Y. Yoshida, H. Nakamura, D. Book, T. Kagotami, and M. Homma, "Barium M-type ferrite as an electromagnetic microwave absorber in the GHz range," Mater. Trans. JIM 39(10), 1080-1083 (1998).

${ }^{22}$ A. Verma, R. G. Mendiratta, T. C. Goel, and D. C. Dube, "Microwave studies on strontium ferrite based absorbers," J. Electroceram. 8, 203-208 (2002).

${ }^{23}$ S. Tyagi, H. B. Baskey, R. C. Agarwala, V. Agarwala, and T. C. Shami, "Development of hard/soft ferrite nanocomposite for enhanced microwave absorption," Ceram. Int. 37, 2631-2641 (2011).

${ }^{24}$ O. Masala, D. Hoffman, N. Sundaram, K. Page, T. Proffen, G. Lawes, and R. Seshadri, "Preparation of magnetic spinel ferrite core/shell nanoparticles: soft ferrite on hard ferrites and vice versa," Solid State Sci. 8, 1015-1022 (2006).

${ }^{25}$ T. Maeda, S. Sugimoto, T. Kagotani, N. Tezuka, and K. Inomata, "Effect of soft/hard exchange interaction on natural resonance frequency and electromagnetic wave absorption of the rare earth-iron-boron compounds," J. Magn. Magn. Mater. 281, 195-205 (2004).

${ }^{26}$ J. R. Liu, M. Itoh, and K. I. Machida, "Magnetic and electromagnetic wave absorption properties of alpha-Fe/Z-type Ba-ferrite nanocomposites," Appl. Phys. Lett. 88, 062503 (2006).

${ }^{27}$ X. Battle and A. Labarta, J. Phys. D: Appl. Phys. 35, R15-R42 (2002).

${ }^{28}$ S. Y. Zhao, D. K. Lee, C. W. Kim, H. G. Cha, Y. H. Kim, and Y. S. Kang, Bull. Korean Chem. Soc. 27, 237-243 (2006).

${ }^{29}$ J. G. Deng, C. L. He, Y. X. Peng, J. H. Wang, X. P. Long, and P. Li, "Magnetic and conductive $\mathrm{Fe}_{3} \mathrm{O}_{4}$-polyaniline nanoparticles with coreshell structure," Synth. Met. 139, 295-301 (2003).

${ }^{30}$ O. Luukkonen, S. I. Maslovski, and S. A. Tretyakov, "A stepwise nicolson-ross-weir-based material parameter extraction method," IEEE Antennas Wirel. Propag. Lett. 10, 1295-1298 (2011).

${ }^{31}$ K. K. Karkkainen, A. H. Sihvola, and K. I. Nikoskinen, "Effective permittivity of mixtures: numerical validation by the FDTD method," IEEE Trans. Geosci. Remote Sens. 38(3), 1303-1308 (2000).

${ }^{32}$ M. Y. Koledintseva, S. K. Patil, R. W. Schwartz, W. Huebner, K. N. Rozanov, S. Jianxiang, and J. Chen, "Prediction of effective permittivity 
of diphasic dielectrics as a function of frequency," IEEE Trans. Dielectr. Electr. Insul. 16(3), 793-808 (2009).

${ }^{33} \mathrm{~K}$. W. Whites and W. Feng, "Effects of particle shape on the effective permittivity of composite materials with measurements for lattices of cubes," IEEE Trans. Microwave Theory Tech. 50(7), 1723-1729 (2002).

${ }^{34} \mathrm{C}$. Brosseau and P. Talbot, "Effective permittivity of nanocomposite powder compacts," IEEE Trans. Dielectr. Electr. Insul. 11(5), 819-832 (2004).

${ }^{35}$ J. V. Mantese, A. L. Micheli, D. F. Dungan, R. G. Geyer, J. Baker-Jarvis, and J. Grosvenor, "Applicability of effective medium theory to ferroelectric/ferrimagnetic composites with composition and frequency-dependent complex permittivities and permeabilities," J. Appl. Phys. 79(3), 1655-1660 (1996).

${ }^{36} \mathrm{H}$. Looyenga, Physica 31, 401-406 (1965).

${ }^{37}$ J. Zhang, J. Chen, and Z. Wang, Mater. Lett. 61, 1629-1632 (2007).

${ }^{38}$ J. Sun, S. Zhou, P. Hou, Y. Yang, J. Weng, X. Li, and M. Li, J. Biomed. Mater. Res. Part A 80A, 333-341 (2007).
${ }^{39} \mathrm{M}$. Wan, J. Li, and S. Li, "Microtubules of polyaniline as new microwave absorbent materials," Polym. Adv. Technol. 12, 651-657 (2001).

${ }^{40}$ M. Ferrari and L. Lutterotti, J. Appl. Phys. 76, 7246-7255 (1994).

${ }^{41}$ R. Skomski, "Method for the simultaneous determination of anisotropic residual stresses and texture by x-ray diffraction," Simple Models of Magnetism (Oxford University Press, Oxford, United Kingdom, 2008).

${ }^{42}$ J. Smit and H. P. J. Wijn, Ferrites (Philips Technical Library, Eindhoven, NL, 1959).

${ }^{43}$ D. Hasegawa, H. Yang, T. Ogawa, and M. Takahashi, "Challenge of ultra high frequency limit of permeability for magnetic nanoparticle assembly with organic polymer-Application of superparamagnetism," J. Magn. Magn. Mater. 321, 746-749 (2009).

${ }^{44}$ N.-N. Song, H.-T. Yang, H.-L. Liu, X. Ren, H.-F. Ding, X.-Q. Zhang, and Z.-H. Cheng, "Exceeding natural resonance frequency limit of monodisperse $\mathrm{Fe}_{3} \mathrm{O}_{4}$ nanoparticles via superparamagnetic relaxation," Sci. Rep. 3, 3161 (2013). 